\title{
LOGARITHMIC COMBINATORIAL DIFFERENTIALS
}

\author{
DANIEL SCHEPLER
}

\begin{abstract}
Given a morphism $X \rightarrow S$ of fine $\log$ schemes, we develop a geometric description of the sheaves of higher-order differentials $\Omega_{X / S}^{n}$ for $n>1$, as well as a definition of the de Rham complex in terms of this description.
\end{abstract}

\section{Introduction}

Given a smooth morphism $X \rightarrow S$ of schemes, it is standard to define $\Omega_{X / S}^{1}:=$ $I / I^{2}$, where $I$ is the ideal sheaf of the diagonal in $X \times{ }_{S} X$. One normally then defines $\Omega_{X / S}^{q}:=\bigwedge^{q} \Omega_{X / S}^{1}$ for $q>1$. On the other hand, in [1], Breen and Messing give an alternate definition of $\Omega_{X / S}^{q}$ extending the geometric definition of $\Omega_{X / S}^{1}$. This paper was inspired by similar definitions introduced by A. Kock in his study of synthetic differential geometry [3], which in turn was an attempt to transpose the methods in algebraic geometry, due to Grothendieck and others, of studying the concept of infinitesimally close points to the setting of $C^{\infty}$-manifolds.

For simplicity, let us assume that 2 is invertible on $S$. Let $\Delta_{X / S}^{n}:=X \times{ }_{S} X \times{ }_{S} \cdots \times{ }_{S}$ $X$ be the $n+1$-fold product, with the factors indexed from 0 to $n$. For $0 \leq i, j \leq n$, let $I_{i j}$ be ideal of $\mathscr{O}_{\Delta_{X / S}^{n}}$ defining the partial diagonal $\left\{\left(x_{0}, \ldots, x_{n}\right) \in \Delta_{X / S}^{n}: x_{i}=x_{j}\right\}$. Now let $\Delta_{X / S}^{(n)}$ denote the closed subscheme of $\Delta_{X / S}^{n}$ defined by $\sum_{0 \leq i, j \leq n} I_{i j}^{2}$, and $\tilde{I}_{i j}$ the image of $I_{i j}$ in $\mathscr{O}_{\Delta_{X / S}^{(n)}}$. Then

$$
\prod_{i=1}^{n} \tilde{I}_{i-1, i}=\bigcap_{i=1}^{n} \tilde{I}_{i-1, i}=\prod_{i=1}^{n} \tilde{I}_{0 i}=\bigcap_{i=1}^{n} \tilde{I}_{0 i}=\bigcap_{0 \leq i, j \leq n} \tilde{I}_{i j},
$$

and this common ideal, considered as an $\mathscr{O}_{X}$-module via any of the $n+1$ projections $\Delta_{X / S}^{(n)} \rightarrow X$, is canonically isomorphic to $\Omega_{X / S}^{n}$. (In the general case, this construction instead gives the $n$th antisymmetric power of $\Omega_{X / S}^{1}$.)

Our first observation is that in the general case, we can fix this discrepancy by starting with the divided power envelope $D(n)$ of the diagonal in $\Delta_{X / S}^{n}$. In other words, if we let $\Delta_{X / S}^{[n]}$ be the closed subscheme of $D(n)$ defined by $\sum_{0 \leq i, j \leq n} \bar{I}_{i j}^{[2]}$, and $\tilde{I}_{i j}$ the image of $\bar{I}_{i j}$ in $\mathscr{O}_{\Delta_{X / S}^{[n]}}$, then the five ideals above are once again equal, and are canonically isomorphic to $\Omega_{X / S}^{n}$. (In [1], Breen and Messing corrected the discrepancy by expanding $\sum_{0 \leq i, j \leq n} I_{i j}^{2}$ in a non-symmetric way.)

Log geometry provides a convenient language for discussing topics related to compactification and singularities. Recall that a pre-log scheme $X$ is a scheme $X$ equipped

Received by the editors February 14, 2008. 
with a sheaf of commutative monoids $\mathscr{M}_{X}$ and a morphism $\alpha_{X}: \mathscr{M}_{X} \rightarrow \mathscr{O}_{X}^{\times}$, where $\mathscr{O}_{X}^{X}$ is the multiplicative monoid of $\mathscr{O}_{X}$. (Note that we use additive notation for $\mathscr{M}_{X}$, thus considering $m \in \mathscr{M}_{X}$ to be a logarithm of $\alpha(m)$, and considering $\alpha$ to be an exponentation map.) This is a log scheme if the induced morphism $\alpha_{X}^{-1}\left(\mathscr{O}_{X}^{*}\right) \rightarrow \mathscr{O}_{X}^{*}$ is an isomorphism. A log scheme is called fine if locally the log structure is induced by a pre-log structure $P \rightarrow \mathscr{O}_{X}^{\times}$where $P$ is the constant sheaf of a finitely-generated integral monoid. Given a morphism $X \rightarrow S$ of $\log$ scheme, Kato [2] defines a universal sheaf of relative log differentials $\Omega_{X / S}^{1}$ with a $\log$ derivation $(d, d \log ):\left(\mathscr{O}_{X}, \mathscr{M}_{X}\right) \rightarrow \Omega_{X / S}^{1}$. This means that $d: \mathscr{O}_{X} \rightarrow \Omega_{X / S}^{1}$ is an $\mathscr{O}_{S}$-derivation, $d \log : \mathscr{M}_{X} \rightarrow \Omega_{X / S}^{1}$ is an additive map annihilating the image of $\mathscr{M}_{S}$, and for $m \in \mathscr{M}_{X}$, we have

$$
d \alpha(m)=\alpha(m) d \log m .
$$

For example, suppose $X$ is a smooth scheme over a field $k$, and $D$ is a divisor with normal crossings on $X$. Let $Y:=X \backslash D$, with open immersion $i: Y \rightarrow X$. We then define $\mathscr{M}_{X}:=i_{*} \mathscr{O}_{Y}^{*} \cap \mathscr{O}_{X}$, with $\alpha_{X}$ the natural inclusion map. This defines a $\log$ scheme, and the sheaf of $\log$ differentials $\Omega_{X / k}^{1}$ is exactly the classical sheaf $\Omega_{X / k}^{1}(\log D)$ of differentials with $\log$ poles along $D$.

Our aim in this paper is to extend Breen and Messing's theory to give an intrinsic geometric description of $\wedge^{n} \Omega_{X / S}^{1}$ for $n>1$ in the case of log schemes. Thus, consider a morphism $X \rightarrow S$ of fine $\log$ schemes. (Note that we do not require this morphism to be $\log$ smooth.) Again, let $\Delta_{X / S}^{n}:=X \times_{S} \cdots \times_{S} X$ be the $n+1$-fold product. Then there exists a right universal $\log$ scheme $D(n)$ with an exact closed immersion $X \rightarrow D(n)$ defined by a PD ideal on $D(n)$, and a morphism $D(n) \rightarrow \Delta_{X / S}^{n}$, factoring the diagonal morphism $X \rightarrow \Delta_{X / S}^{n}[2]$. Again, let $\Delta_{X / S}^{[n]}$ be the closed subscheme of $D(n)$ defined by the ideal $\sum_{0 \leq i, j \leq n} \bar{I}_{i j}^{[2]}$, where $\bar{I}_{i j}$ is the ideal of the partial diagonal $\left\{x_{i}=x_{j}\right\}$ in $\Delta(n)$, and $\tilde{I}_{i j}$ the image of $\bar{I}_{i j}$ in $\mathscr{O}_{\Delta_{X / S}^{[n]}}$. Then we will prove that in this more general case, once again the five ideals above are equal and are canonically isomorphic to $\Omega_{X / S}^{n}$. The proof we give here is an improvement on the proof given in [1].

In terms of this description, the de Rham complex becomes particularly simple, in the form of an Alexander-Spaniel complex. First, for $m, n \geq 0$, consider $\Delta_{X / S}^{m}$ as a scheme over $X$ via the last projection, and $\Delta_{X / S}^{n}$ as a scheme over $X$ via the first projection. Then we have a morphism

$$
\begin{aligned}
\Delta_{X / S}^{m+n} & \rightarrow \Delta_{X / S}^{m} \times_{X} \Delta_{X / S}^{n}, \\
\left(x_{0}, \ldots, x_{m}, \ldots, x_{m+n}\right) & \mapsto\left(\left(x_{0}, \ldots, x_{m}\right),\left(x_{m}, \ldots, x_{m+n}\right)\right) .
\end{aligned}
$$

This induces a map $\Delta_{X / S}^{[m+n]} \rightarrow \Delta_{X / S}^{[m]} \times_{X} \Delta_{X / S}^{[n]}$, which in turn induces the wedge product. Similarly, given $n \geq 0$ and $0 \leq i \leq n+1$, define $d_{i}: \Delta_{X / S}^{n+1} \rightarrow \Delta_{X / S}^{n}$ to be the map which forgets the $i$ th component. This induces maps $d_{i}: \Delta_{X / S}^{[n+1]} \rightarrow \Delta_{X / S}^{[n]}$, and the differential $d: \Omega_{X / S}^{n} \rightarrow \Omega_{X / S}^{n+1}$ is induced by

$$
d_{0}^{*}-d_{1}^{*}+\cdots+(-1)^{n+1} d_{n+1}^{*}: \mathscr{O}_{\Delta_{X / S}^{[n]}} \rightarrow \mathscr{O}_{\Delta_{X / S}^{[n+1]}} .
$$


Finally, suppose $X \rightarrow S$ is log smooth. We observe that each $\Delta_{X / S}^{[n]}$ is an object in the $\log$ crystalline site of $X$ over $S$, and each $d_{i}$ is a morphism in this site. Therefore, given a crystal $E$ on this site, which corresponds to a module with quasi-nilpotent connection $\left(E_{X}, \nabla\right)$, we have transition maps $\theta_{d_{i}}: E_{\Delta_{X / S}^{[n]}} \rightarrow E_{\Delta_{X / S}^{[n+1]}}$. Here $E_{\Delta_{X / S}^{[n]}} \simeq$ $E_{X} \otimes_{\mathscr{O}_{X}} \mathscr{O}_{\Delta_{X / S}^{[n]}}$ via the isomorphism $\theta_{\pi_{0}}: \pi_{0}^{*} E_{X} \rightarrow E_{\Delta_{X / S}^{[n]}}$. We will show that the differential

$$
\nabla: E_{X} \otimes_{\mathscr{O}_{X}} \Omega_{X / S}^{n} \rightarrow E_{X} \otimes_{\mathscr{O}_{X}} \Omega_{X / S}^{n+1}
$$

in the de Rham complex of $\left(E_{X}, \nabla\right)$ is induced by

$$
\theta_{d_{0}}-\theta_{d_{1}}+\cdots+(-1)^{n+1} \theta_{d_{n+1}}: E_{X} \otimes_{\mathscr{O}_{X}} \mathscr{O}_{\Delta_{X / S}^{[n]}} \rightarrow E_{X} \otimes_{\mathscr{O}_{X}} \mathscr{O}_{\Delta_{X / S}^{[n+1]}}
$$

\section{Combinatorial Differentials}

1.1. Local Construction. We begin in this section with a simplified situation: suppose $A$ is a ring, $B$ an $A$-algebra, and $Q \rightarrow P$ a morphism of finitely-generated integral monoids with compatible maps $Q \rightarrow A$ and $P \rightarrow B$. Let $S:=\operatorname{Spec} A$, $X:=\operatorname{Spec} B$, with the $\log$ structures induced by $Q$ and $P$, respectively. Now let $\Delta_{X / S}^{n}:=X \times_{S} X \times_{S} \cdots \times_{S} X$ be the $n+1$-fold product, with the factors indexed from 0 to $n$. In other words, $\Delta_{X / S}^{n}=\operatorname{Spec}\left(B_{n}\right)$, where $B_{n}:=B \otimes_{A} \cdots \otimes_{A} B$, with $\log$ structure induced by $P_{n}$, the quotient of $P \oplus P \oplus \cdots \oplus P$ by the congruence generated by

$$
(q, 0, \cdots, 0) \equiv(0, q, \cdots, 0) \equiv \cdots \equiv(0,0, \cdots, q)
$$

for $q \in Q$. Then $P_{n}^{\mathrm{gp}}$ is the quotient of $P^{\mathrm{gp}} \oplus P^{\mathrm{gp}} \oplus \cdots \oplus P^{\mathrm{gp}}$ by $\left\{\left(q_{0}, q_{1}, \ldots, q_{n}\right) \in\right.$ $\left.Q^{\mathrm{gp}} \oplus \cdots \oplus Q^{\mathrm{gp}}: q_{0}+\cdots+q_{n}=0\right\}$.

Now the diagonal map $X \rightarrow \Delta_{X / S}^{n}$ corresponds to the product map $B_{n} \rightarrow B$, and it has a chart given by the sum map $P_{n} \rightarrow P$. Now let

$$
P_{n}^{\prime}:=\left\{\left(p_{0}, p_{1}, \ldots, p_{n}\right) \in P_{n}^{\mathrm{gp}}: p_{0}+p_{1}+\ldots+p_{n} \in P\right\},
$$

$B_{n}^{\prime}:=B_{n} \otimes_{\mathbb{Z}\left[P_{n}\right]} \mathbb{Z}\left[P_{n}^{\prime}\right]$, and $Z_{n}:=$ Spec $B_{n}^{\prime}$ with the log structure induced by $P_{n}^{\prime}$. (For $p \in P$, we will use the notation $e^{p}$ for the corresponding element of $\mathbb{Z}[P]$, in order to avoid confusion between addition in $P$ and addition in $\mathbb{Z}[P]$.) Then the map $X \rightarrow Z_{n}$ corresponding to the sum map $P_{n}^{\prime} \rightarrow P$ is an exact closed immersion, and the map $Z_{n} \rightarrow \Delta_{X / S}^{n}$ corresponding to the inclusion $P_{n} \hookrightarrow P_{n}^{\prime}$ is log étale. Therefore, $Z_{n}$ may be used as the basis for constructing the log infinitesimal neighborhoods and the divided power envelope of $X$ in $\Delta_{X / S}^{n}$ [2]. (Recall that a map $g: Q \rightarrow P$ of integral monoids is exact if $\left(g^{\mathrm{gp}}\right)^{-1}(P)=Q$, and a morphism $X \rightarrow S$ of fine $\log$ schemes is exact if for every point $x \in X$ with image $s \in S, \mathscr{M}_{S, s} \rightarrow \mathscr{M}_{X, x}$ is exact. A log closed immersion $f: X \rightarrow Y$ is exact if and only if it is strict, i.e. $f^{*} \mathscr{M}_{Y} \rightarrow \mathscr{M}_{X}$ is an isomorphism, where $f^{*} \mathscr{M}_{Y}$ is the log structure induced by $f^{-1} \mathscr{M}_{Y}$.)

For notation, let $\pi_{i}^{*}: P \rightarrow P_{n}^{\prime}$ be the $i$ th inclusion map, corresponding to the $i$ th projection $\pi_{i}: Z_{n} \rightarrow X$. Now for each pair $i, j$ with $0 \leq i, j \leq n$, we have a closed immersion $m_{i j}: Z_{n-1} \rightarrow Z_{n}$ corresponding to the map $\mu_{i j}: B_{n}^{\prime} \rightarrow B_{n-1}^{\prime}$,

$$
\begin{gathered}
\left(y_{0} \otimes \cdots \otimes y_{i} \otimes \cdots \otimes y_{j} \otimes \cdots \otimes y_{n}\right) \otimes e^{\left(p_{0}, \ldots, p_{i}, \ldots, p_{j}, \ldots, p_{n}\right)} \mapsto \\
\left(y_{0} \otimes \cdots \otimes y_{i} y_{j} \otimes \cdots \otimes \hat{y}_{j} \otimes \cdots \otimes y_{n}\right) \otimes e^{\left(p_{0}, \ldots, p_{i}+p_{j}, \ldots, \hat{p}_{j}, \ldots, p_{n}\right)} .
\end{gathered}
$$


Let $I_{i j} \subseteq \mathscr{O}_{Z_{n}}$ be the ideal sheaf defining this closed immersion, and $\Delta_{X / S}^{(n)}$ the closed subscheme of $Z_{n}$ defined by $\sum_{0 \leq i, j \leq n} I_{i j}^{2}$. It is easy to see that $I_{i j}$ is generated by elements of the form

for $p \in P^{\mathrm{gp}}$ and

$$
\delta^{i, j} p:=1 \otimes\left(e^{\pi_{j}^{*} p-\pi_{i}^{*} p}-1\right) \in B_{n}^{\prime}
$$

$$
d^{i, j} y:=\left(\pi_{j}^{*} y-\pi_{i}^{*} y\right) \otimes 1
$$

for $y \in B$. Let $\tilde{I}_{i j}$ be the image of $I_{i j}$ in $\mathscr{O}_{\Delta_{X / S}^{(n)}}$.

We first note the following for future reference:

Lemma 1.1. Let $0 \leq i, j, k, \ell \leq n$.

(1) Assume $i<j$ and $k<\ell$. Then $\mu_{i j}\left(I_{k \ell}\right)=0$ if $i=k$ and $j=\ell$; otherwise, $\mu_{i j}\left(I_{k \ell}\right)=I_{k^{\prime} \ell^{\prime}}$, where

$$
k^{\prime}= \begin{cases}k, & k<j \\ i, & k=j \\ k-1, & k>j,\end{cases}
$$

and similarly for $\ell^{\prime}$. Hence $\mu_{i j}$ gives a well-defined map $\mathscr{O}_{\Delta_{X / S}^{(n)}} \rightarrow \mathscr{O}_{\Delta_{X / S}^{(n-1)}}$, and the same is true with $\tilde{I}_{k \ell}$ and $\tilde{I}_{k^{\prime} \ell^{\prime}}$ in place of $I_{k \ell}$ and $I_{k^{\prime} \ell^{\prime}}$.

(2) $I_{i \ell} \subseteq I_{i j}+I_{j \ell}$.

Proof. The first statement follows from the fact that $\mu_{i j}$ acts the same on the generators $d^{k, \ell} y$ and $\delta^{k, \ell} p$ of $I_{k \ell}$.

For the second statement, note that $d^{i, \ell} y=d^{i, j} y+d^{j, \ell} y$ for $y \in B$. Similarly, since $1+\delta^{i, j} p=1 \otimes e^{\pi_{j}^{*} p-\pi_{i}^{*} p}$, for $p \in P^{\mathrm{gp}}$ we have

$$
1+\delta^{i, \ell} p=\left(1+\delta^{i, j} p\right)\left(1+\delta^{j, \ell} p\right) .
$$

Therefore, $\delta^{i, \ell} p=\delta^{i, j} p+\delta^{j, \ell} p+\left(\delta^{i, j} p\right)\left(\delta^{j, \ell} p\right) \in J_{i j}+J_{j \ell}$ also.

Let $\Omega_{X / S}^{(n)}$ be the $n$th antisymmetric product of $\Omega_{X / S}^{1}$. We first define a map $\bigcap_{i=1}^{n} \tilde{I}_{0 i} \rightarrow \Omega_{X / S}^{(n)}$.

Proposition 1.2. There exists a unique A-linear map $\Psi_{n}: B_{n}^{\prime} \rightarrow \Gamma\left(X, \Omega_{X / S}^{(n)}\right)$ such that for $y_{0}, \ldots, y_{n} \in B,\left(p_{0}, \ldots, p_{n}\right) \in P_{n}^{\prime}$, we have

$$
\begin{aligned}
& \Psi_{n}\left[\left(y_{0} \otimes \cdots \otimes y_{n}\right) \otimes e^{\left(p_{0}, \ldots, p_{n}\right)}\right]= \\
& \quad y_{0} \alpha\left(p_{0}+p_{1}+\cdots+p_{n}\right)\left(d y_{1}+y_{1} d \log p_{1}\right) \tilde{\wedge} \cdots \tilde{\wedge}\left(d y_{n}+y_{n} d \log p_{n}\right) .
\end{aligned}
$$

(Here $\tilde{\wedge}$ denotes the product in the antisymmetric product algebra $\left.\Omega_{X / S}^{(\cdot)} \cdot{ }^{\cdot}\right)$

Proof. The uniqueness is clear. To see the map is well-defined, we have several things to check:

- The above expression is $A$-multilinear in the variables $y_{0}, y_{1}, \ldots, y_{n}$.

This is clear from the $A$-linearity of $d$.

- The expression above is independent of the choice of $p_{0}, \ldots, p_{n} \in P^{g p}$.

This follows from the fact that $p_{0}+\cdots+p_{n} \in P$ is well-defined, and the fact that $d \log$ induces a well-defined map $P^{\mathrm{gp}} / Q^{\mathrm{gp}} \rightarrow \Omega_{X / S}^{1}$. 
- For $p^{\prime} \in P$,

$$
\begin{gathered}
\Psi_{n}\left[\left(y_{0} \otimes \cdots \otimes y_{i} \alpha\left(p^{\prime}\right) \otimes \cdots \otimes y_{n}\right) \otimes e^{\left(p_{0}, \ldots, p_{i}, \ldots, p_{n}\right)}\right]= \\
\Psi_{n}\left[\left(y_{0} \otimes \cdots \otimes y_{i} \otimes \cdots \otimes y_{n}\right) \otimes e^{\left(p_{0}, \ldots, p_{i}+p^{\prime}, \ldots, p_{n}\right)}\right] .
\end{gathered}
$$

For $i=0$, this is clear. Otherwise, for $i>0$, this follows from the formula

$$
d\left(y_{i} \alpha\left(p^{\prime}\right)\right)=y_{i} d\left(\alpha\left(p^{\prime}\right)\right)+\alpha\left(p^{\prime}\right) d y_{i}=\alpha\left(p^{\prime}\right)\left[d y_{i}+y_{i} d \log p^{\prime}\right] .
$$

Remark 1.3. In the case of trivial $\log$ structure, i.e. $P=0$, the formula for $\Psi_{n}$ reduces to

$$
\Psi_{n}\left(y_{0} \otimes y_{1} \otimes \cdots \otimes y_{n}\right)=y_{0} d y_{1} \tilde{\wedge} \cdots \tilde{\wedge} d y_{n} .
$$

This is the isomorphism commonly used in synthetic differential geometry, for example in [3].

Also note that if in fact $p_{0}, \ldots, p_{n} \in P$, then

$$
\left(y_{0} \otimes y_{1} \otimes \cdots \otimes y_{n}\right) \otimes e^{\left(p_{0}, p_{1}, \ldots, p_{n}\right)}=\left(y_{0} \alpha\left(p_{0}\right) \otimes y_{1} \alpha\left(p_{1}\right) \otimes \cdots \otimes y_{n} \alpha\left(p_{n}\right)\right) \otimes 1,
$$

and in this case the formula for $\Psi_{n}$ agrees with

$$
y_{0} \alpha\left(p_{0}\right) d\left(y_{1} \alpha\left(p_{1}\right)\right) \tilde{\wedge} \cdots \tilde{\wedge} d\left(y_{n} \alpha\left(p_{n}\right)\right) .
$$

Thus we may view the given formula for $\Psi_{n}$ as a natural generalization of the simpler formula from the case of trivial log structure.

Proposition 1.4. The map $\Psi_{n}$ annihilates $I_{i j}^{2}$ for each pair $0 \leq i, j \leq n$.

Proof. We first check the case $i=0$. In this case, since $d\left(y_{j} y\right)=y_{j} d y+y d y_{j}$, it is straightforward to calculate that for $x \in B_{n}^{\prime}, y \in B$, we have

$$
\Psi_{n}\left(x d^{0, j} y\right)=(-1)^{j-1} d y \tilde{\wedge} \Psi_{n-1}\left(\mu_{0, j} x\right) .
$$

Therefore, if $x \in I_{0 j}$, then $\Psi_{n}\left(x d^{0, j} y\right)=0$. Similarly, for $p \in P^{\mathrm{gp}}$,

$$
\Psi_{n}\left(x \delta^{0, j} p\right)=(-1)^{j-1} d \log p \tilde{\wedge} \Psi_{n-1}\left(\mu_{0, j} x\right),
$$

so again if $x \in I_{0 j}$, then $\Psi_{n}\left(x \delta^{0, j} p\right)=0$.

Now for the general case, by symmetry assume $i<j$. We observe that $d^{i, j} y=$ $d^{0, j} y-d^{0, i} y$. Thus, if $y, y^{\prime} \in B$, then

$$
\left(d^{i, j} y\right)\left(d^{i, j} y^{\prime}\right) \equiv-\left[\left(d^{0, i} y\right)\left(d^{0, j} y^{\prime}\right)+\left(d^{0, i} y^{\prime}\right)\left(d^{0, j} y\right)\right] \quad\left(\bmod J_{0 i}^{2}+J_{0 j}^{2}\right) .
$$

However, since $\mu_{0 j}\left(x\left(d^{0, i} y^{\prime}\right)\right)=\left(\mu_{0 j} x\right)\left(d^{0, i} y\right)$ for $x \in B_{n}^{\prime}$, we have

$$
\begin{aligned}
\Psi_{n}\left(x\left(d^{0, i} y\right)\left(d^{0, j} y^{\prime}\right)\right) & =(-1)^{j-1} d y^{\prime} \tilde{\wedge} \Psi_{n-1}\left(\left(\mu_{0 j} x\right)\left(d^{0, i} y\right)\right) \\
& =(-1)^{i+j} d y^{\prime} \tilde{\wedge} d y \tilde{\wedge} \Psi_{n-2}\left(\mu_{0 i} \mu_{0 j} x\right) .
\end{aligned}
$$

Therefore, for $x \in B_{n}^{\prime}$,

$$
\Psi_{n}\left(x\left(d^{i, j} y\right)\left(d^{i, j} y^{\prime}\right)\right)=(-1)^{i+j+1}\left(d y^{\prime} \tilde{\wedge} d y+d y \tilde{\wedge} d y^{\prime}\right) \tilde{\wedge} \Psi_{n-2}\left(\mu_{0 i} \mu_{0 j} x\right)=0 .
$$

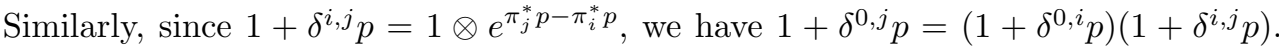
Multiplying both sides by $1-\delta^{0, i} p$, this implies

$$
1+\delta^{i, j} p \equiv\left(1+\delta^{0, j} p\right)\left(1-\delta^{0, i} p\right) \quad\left(\bmod J_{0 i}^{2}+J_{0 j}^{2}\right),
$$


so $\delta^{i, j} p \equiv \delta^{0, j} p-\delta^{0, i} p-\left(\delta^{0, j} p\right)\left(\delta^{0, i} p\right)$. Therefore,

$$
\left(\delta^{i, j} p\right)\left(d^{i, j} y\right) \equiv-\left[\left(\delta^{0, i} p\right)\left(d^{0, j} y\right)+\left(d^{0, i} y\right)\left(\delta^{0, j} p\right)\right] \quad\left(\bmod J_{0 i}^{2}+J_{0 j}^{2}\right)
$$

and

$$
\left(\delta^{i, j} p\right)\left(\delta^{i, j} p^{\prime}\right) \equiv-\left[\left(\delta^{0, i} p\right)\left(\delta^{0, j} p^{\prime}\right)+\left(\delta^{0, i} p^{\prime}\right)\left(\delta^{0, j} p\right)\right] \quad\left(\bmod J_{0 i}^{2}+J_{0 j}^{2}\right) .
$$

From these formulas, the proof that $\Psi$ annihilates $x\left(\delta^{i, j} p\right)\left(d^{i, j} y\right)$ and $x\left(\delta^{i, j} p\right)\left(\delta^{i, j} p^{\prime}\right)$ proceeds as before.

Therefore, since $\Omega_{X / S}^{(n)}$ is a quasi-coherent $\mathscr{O}_{X}$-module, $\Psi_{n}$ induces a map $\Psi_{n}$ : $\mathscr{O}_{\Delta_{X / S}^{(n)}} \rightarrow \Omega_{X / S}^{(n)}$, which we will restrict to the ideal $\bigcap_{j=1}^{n} \tilde{I}_{0 j}$ of $\mathscr{O}_{\Delta_{X / S}^{(n)}}$. We now turn to defining a map in the other direction.

Proposition 1.5. For each $i$ with $0<i \leq n$, there is a unique B-linear map $\phi_{i}$ : $\Omega_{X / S}^{1} \rightarrow \tilde{I}_{0 i}$ such that $\phi_{i}(d y)=d^{0, i} y$ for $y \in B$ and $\phi_{i}(d \log p)=\delta^{0, i} p$ for $p \in P^{\mathrm{gp}}$. (Here we consider $\tilde{I}_{0 i}$ to be a $B$-module via $\pi_{0}^{*}$.)

Proof. By the universal property of $\Omega_{X / S}^{1}$, we need only check that $(D, \delta):\left(B, P^{g p}\right) \rightarrow$ $\tilde{I}_{0 i}$ defined by $D y=d^{0, i} y$ and $\delta p=\delta^{0, i} p$ is a $\log$ derivation over $A$. However, $D$ is clearly $A$-linear, and since

$$
\left(d^{0, i} y\right)\left(d^{0, i} y^{\prime}\right)=d^{0, i}\left(y y^{\prime}\right)-\left(\pi_{0}^{*} y\right) d^{0, i} y^{\prime}-\left(\pi_{0}^{*} y^{\prime}\right) d^{0, i} y \in I_{0 i}^{2},
$$

$D$ is also a derivation. Similarly, for $p \in P$, we have $d^{0, i}(\alpha(p))=\left(\pi_{0}^{*} \alpha(p)\right) \delta^{0, i} p$. Finally, to see that $\delta$ is additive, since $1+\delta^{0, i} p=1 \otimes e^{\pi_{i}^{*} p-\pi_{0}^{*} p}$, we have

$$
1+\delta^{0, i}\left(p+p^{\prime}\right)=\left(1+\delta^{0, i} p\right)\left(1+\delta^{0, i} p^{\prime}\right)=1+\delta^{0, i} p+\delta^{0, i} p^{\prime}+\left(\delta^{0, i} p\right)\left(\delta^{0, i} p^{\prime}\right) .
$$

Therefore, $\delta^{0, i}\left(p+p^{\prime}\right) \equiv \delta^{0, i} p+\delta^{0, i} p^{\prime}\left(\bmod J_{0 i}^{2}\right)$.

Proposition 1.6. There is a unique map $\Phi_{n}: \Omega_{X / S}^{(n)} \rightarrow \prod_{j=1}^{n} \tilde{J}_{0 j}$ such that for $\omega_{1}, \omega_{2}, \ldots, \omega_{n} \in \Omega_{X / S}^{1}$,

$$
\Phi_{n}\left(\omega_{1} \tilde{\wedge} \omega_{2} \tilde{\wedge} \cdots \tilde{\wedge} \omega_{n}\right)=\phi_{1}\left(\omega_{1}\right) \phi_{2}\left(\omega_{2}\right) \cdots \phi_{n}\left(\omega_{n}\right) .
$$

Proof. Since the formula above is clearly multilinear in $\omega_{1}, \ldots, \omega_{n}$, we need only check it is antisymmetric. We claim that in fact, for $\omega, \tau \in \Omega_{X / S}^{1}, \phi_{i}(\omega) \phi_{j}(\tau)+\phi_{i}(\tau) \phi_{j}(\omega)=$ 0 in $\mathscr{O}_{\Delta_{X / S}^{(n)}}$. To see this, we refer again to the formulas (1.1) through (1.3). Thus, if $\omega=d y$ and $\tau=d y^{\prime}$, then by (1.1),

$$
\left(d^{0, i} y\right)\left(d^{0, j} y^{\prime}\right)+\left(d^{0, i} y^{\prime}\right)\left(d^{0, j} y\right) \equiv-\left(d^{i, j} y\right)\left(d^{i, j} y^{\prime}\right) \quad\left(\bmod J_{0 i}^{2}+J_{0 j}^{2}\right),
$$

so $\phi_{i}(\omega) \phi_{j}(\tau)+\phi_{i}(\tau) \phi_{j}(\omega) \in J_{0 i}^{2}+J_{0 j}^{2}+J_{i j}^{2}$. Similarly, for the cases $\omega=d \log p$, $\tau=d y$ and $\omega=d \log p, \tau=d \log p^{\prime}$, we use the corresponding formulas (1.2) and (1.3).

We now show the two maps defined above are inverses. 
Theorem 1.7. (1) The composition

$$
\Omega_{X / S}^{(n)} \stackrel{\Phi_{n}}{\longrightarrow} \prod_{j=1}^{n} \tilde{J}_{0 j} \stackrel{\Psi_{n}}{\longrightarrow} \Omega_{X / S}^{(n)}
$$

is the identity on $\Omega_{X / S}^{(n)}$.

(2) The composition

$$
\bigcap_{j=1}^{n} \tilde{J}_{0 j} \stackrel{\Psi_{n}}{\longrightarrow} \Omega_{X / S}^{(n)} \stackrel{\Phi_{n}}{\longrightarrow} \prod_{j=1}^{n} \tilde{J}_{0 j} \hookrightarrow \bigcap_{j=1}^{n} \tilde{J}_{0 j}
$$

is the identity map on $\bigcap_{j=1}^{n} \tilde{J}_{0 j}$.

Proof. For the first composition, it suffices to check for

$$
\omega=d y_{1} \tilde{\wedge} \cdots \tilde{\wedge} d y_{i} \tilde{\wedge} d \log p_{i+1} \tilde{\wedge} \cdots \tilde{\wedge} d \log p_{n},
$$

for $y_{1}, \ldots, y_{i} \in B, p_{i+1}, \ldots, p_{n} \in P^{\mathrm{gp}}$. However,

$$
\begin{gathered}
\Phi_{n}(\omega)=\sum_{S \subseteq\{1, \ldots, n\}}(-1)^{|S|}\left(y_{0 S} \otimes y_{1 S} \otimes \cdots \otimes y_{i S} \otimes 1 \otimes \cdots \otimes 1\right) \otimes \\
e^{\left(p_{0 S}, 0, \ldots, 0, p_{i+1, S}, \ldots, p_{n S}\right)},
\end{gathered}
$$

where:

- $y_{0 S}=\prod_{1 \leq j \leq i, j \in S} y_{j}$

- $y_{j S}=y_{j}$ if $j \notin S$ and $y_{j S}=1$ if $j \in S, 1 \leq j \leq i$;

- $p_{0 S}=-\sum_{i<j \leq n, j \notin S} p_{j}$;

- $p_{j S}=p_{j}$ if $j \notin S$ and $p_{j S}=0$ if $j \in S, i<j \leq n$.

Therefore,

$$
\Psi_{n}\left(\Phi_{n}(\omega)\right)=\sum_{S} y_{0 S} d y_{1 S} \tilde{\wedge} \cdots \tilde{\wedge} d y_{i S} \tilde{\wedge} d \log p_{i+1, S} \tilde{\wedge} \cdots \tilde{\wedge} d \log p_{n S} .
$$

However, if $S \neq \emptyset$, then either $y_{j S}=1$ or $p_{j S}=0$ for some $j \in S$, so the corresponding term is zero. On the other hand, for $S=\emptyset$, the corresponding term is exactly $\omega$.

For the second composition, let $x=\left(y_{0} \otimes \cdots \otimes y_{n}\right) \otimes e^{\left(p_{0}, \ldots, p_{n}\right)} \in B_{n}^{\prime}$. Then since $\pi_{i}^{*} y_{i} \equiv \pi_{0}^{*} y_{i}\left(\bmod J_{0 i}\right)$, we calculate that

$$
\begin{aligned}
\phi_{i}\left(d y_{i}+y_{i} d \log p_{i}\right) & \equiv \pi_{i}^{*} y_{i} \otimes 1-\pi_{0}^{*} y_{i} \otimes 1+\pi_{i}^{*} y_{i} \otimes\left(e^{\pi_{i}^{*} p_{i}-\pi_{0}^{*} p_{i}}-1\right) \\
& =\pi_{i}^{*} y_{i} \otimes e^{\pi_{i}^{*} p_{i}-\pi_{0}^{*} p_{i}}-\pi_{0}^{*} y_{i} \otimes 1 \quad\left(\bmod J_{0 i}^{2}\right) .
\end{aligned}
$$

From this we see that

$$
\Phi_{n} \circ \Psi_{n}=\prod_{j=1}^{n}\left(\mathrm{id}-M_{j}\right)
$$

on $\mathscr{O}_{\Delta_{X / S}^{(n)}}$, where

$$
\begin{array}{r}
M_{j}\left[\left(y_{0} \otimes y_{1} \otimes \cdots \otimes y_{j} \otimes \cdots \otimes y_{n}\right) \otimes e^{\left(p_{0}, p_{1}, \ldots, p_{j}, \ldots, p_{n}\right)}\right]= \\
\left(y_{0} y_{j} \otimes y_{1} \otimes \cdots \otimes 1 \otimes \cdots \otimes y_{n}\right) \otimes e^{\left(p_{0}+p_{j}, p_{1}, \ldots, 0, \ldots, p_{n}\right)} .
\end{array}
$$

However, $M_{j}$ factors through $\mu_{0 j}$, so this implies that $\Phi_{n} \circ \Psi_{n}=$ id on $\bigcap_{j=1}^{n} \tilde{I}_{0 j}$. 
Corollary 1.8. We have

$$
\bigcap_{j=1}^{n} \tilde{J}_{0 j}=\prod_{j=1}^{n} \tilde{J}_{0 j}=\bigcap_{0 \leq i, j \leq n} \tilde{J}_{i j} \simeq \Omega_{X / S}^{(n)} .
$$

Proof. From the theorem, the inclusion map $\prod_{j=1}^{n} \tilde{J}_{0 j} \hookrightarrow \bigcap_{j=1}^{n} \tilde{J}_{0 j}$ must be surjective, so it is the identity map and the two ideals are equal. Thus $\Phi_{n}$ and $\Psi_{n}$ are inverse isomorphisms between this common ideal and $\Omega_{X / S}^{(n)}$. Now clearly, $\bigcap_{0 \leq i, j \leq n} \tilde{J}_{i j} \subseteq$ $\bigcap_{j=1}^{n} \tilde{J}_{0 j}$. On the other hand, if $0 \leq i, j \leq n$, then $\tilde{J}_{0 j} \subseteq \tilde{J}_{0 i}+\tilde{J}_{i j}$, so $\tilde{J}_{0 i} \tilde{J}_{0 j} \subseteq \tilde{J}_{i j}$. Therefore, $\prod_{j=1}^{n} \tilde{J}_{0 j} \subseteq \bigcap_{0 \leq i, j \leq n} \tilde{J}_{i j}$ also.

We now present another formulation of this ideal which is more useful in certain situations. First, we note that

$$
\begin{aligned}
\tilde{J}_{01} & =\tilde{J}_{01} \\
\tilde{J}_{02} & \subseteq \tilde{J}_{01}+\tilde{J}_{12} ; \\
\tilde{J}_{03} & \subseteq \tilde{J}_{01}+\tilde{J}_{12}+\tilde{J}_{23} ; \\
\quad & \\
\tilde{J}_{0 n} & \subseteq \tilde{J}_{01}+\tilde{J}_{12}+\cdots+\tilde{J}_{n-1, n} .
\end{aligned}
$$

Therefore, $\prod_{j=1}^{n} \tilde{J}_{0 j} \subseteq \prod_{j=1}^{n} \tilde{J}_{j-1, j}$. Similarly, since $\tilde{J}_{j-1, j} \subseteq \tilde{J}_{0, j-1}+\tilde{J}_{0, j}$, the reverse inclusion also holds, and $\prod_{j=1}^{n} \tilde{J}_{0 j}=\prod_{j=1}^{n} \tilde{J}_{j-1, j}$.

In fact, since $d^{0, j} y \equiv d^{j-1, j} y\left(\bmod J_{0, j-1}\right)$ and $\delta^{0, j} p \equiv \delta^{j-1, j} p\left(\bmod J_{0, j-1}\right)$, while $J_{0, j-1} \subseteq J_{01}+J_{12}+\cdots+J_{j-2, j-1}$, we see that

$$
\Phi_{n}\left(\omega_{1} \tilde{\wedge} \cdots \tilde{\wedge} \omega_{n}\right)=\psi_{1}\left(\omega_{1}\right) \cdots \psi_{n}\left(\omega_{n}\right),
$$

where $\psi_{i}(d y)=d^{i-1, i} y$ and $\psi_{i}(d \log p)=\delta^{i-1, i} p$. From this we may calculate that

$$
\Phi_{n} \circ \Psi_{n}=\left(\mathrm{id}-M_{n}^{\prime}\right) \circ \cdots \circ\left(\mathrm{id}-M_{2}^{\prime}\right) \circ\left(\mathrm{id}-M_{1}^{\prime}\right) \text {. }
$$

Here

$$
\begin{aligned}
& M_{j}^{\prime}\left[\left(y_{0} \otimes \cdots \otimes y_{j-1} \otimes y_{j} \otimes \cdots \otimes y_{n}\right) \otimes e^{\left(p_{0}, \ldots, p_{j-1}, p_{j}, \ldots, p_{n}\right)}\right] \\
& \quad=\left(y_{0} \otimes \cdots \otimes y_{j-1} y_{j} \otimes 1 \otimes \cdots \otimes y_{n}\right) \otimes e^{\left(p_{0}, \ldots, p_{j-1}+p_{j}, 0, \ldots, p_{n}\right)} .
\end{aligned}
$$

However, $M_{j}^{\prime}$ factors through $\mu_{j-1, j}$, hence $\Phi_{n} \circ \Psi_{n}=\mathrm{id}$ on $\bigcap_{j=1}^{n} \tilde{J}_{j-1, j}$. From this we conclude that

$$
\bigcap_{j=1}^{n} \tilde{J}_{j-1, j}=\prod_{j=1}^{n} \tilde{J}_{j-1, j}=\bigcap_{j=1}^{n} \tilde{J}_{0 j}=\prod_{j=1}^{n} \tilde{J}_{0 j}=\bigcap_{0 \leq i, j \leq n} \tilde{J}_{i j} .
$$

1.2. Globalization. Now let $X \rightarrow S$ be an arbitrary morphism of fine log schemes, and let $\Delta_{X / S}^{n}:=X \times_{S} X \times_{S} \cdots \times_{S} X$ be the $n+1$-fold product. Let $\tilde{\Delta}_{X / S}^{n}$ be the $\log$ formal neighborhood of the diagonal immersion $\Delta: X \rightarrow \Delta_{X / S}^{n}$. Then for $0 \leq i<j \leq n$, we have a closed immersion $m_{i j}: \Delta_{X / S}^{n-1} \rightarrow \Delta_{X / S}^{n}$ defined by

$$
\begin{array}{r}
m_{i j}\left(x_{0}, \ldots, x_{i}, \ldots, x_{j-1}, x_{j}, \ldots, x_{n-1}\right)= \\
\left(x_{0}, \ldots, x_{i}, \ldots, x_{j-1}, x_{i}, x_{j}, \ldots, x_{n-1}\right) .
\end{array}
$$


This induces a closed immersion $\tilde{\Delta}_{X / S}^{n-1} \rightarrow \tilde{\Delta}_{X / S}^{n}$. Let $J_{i j}$ be the ideal of $\mathscr{O}_{\tilde{\Delta}_{X / S}^{n}}$ defining this closed immersion, and let $\Delta_{X / S}^{(n)}$ be the closed subscheme of $\tilde{\Delta}_{X / S}^{n}$ defined by $\sum_{0 \leq i<j \leq n} J_{i j}^{2}$. Finally, let $\tilde{J}_{i j}$ be the image of $J_{i j}$ in $\mathscr{O}_{\Delta_{X / S}^{(n)}}$.

Theorem 1.9. We have

$$
\bigcap_{j=1}^{n} \tilde{J}_{j-1, j}=\prod_{j=1}^{n} \tilde{J}_{j-1, j}=\bigcap_{j=1}^{n} \tilde{J}_{0 j}=\prod_{j=1}^{n} \tilde{J}_{0 j}=\bigcap_{0 \leq i, j \leq n} \tilde{J}_{i j},
$$

and this ideal is canonically isomorphic to $\Omega_{X / S}^{(n)}$.

Proof. Since the construction above is local with respect to both $X$ and $S$, in proving the first statement we may assume that $X$ and $S$ are affine and that we have a chart $\left(P \rightarrow \mathscr{O}_{X}, Q \rightarrow \mathscr{O}_{S}, Q \rightarrow P\right)$ of the morphism $X \rightarrow S$. Then note that in the construction of the previous section, the ideal $J$ of the diagonal immersion $X \rightarrow \Delta_{X / S}^{n}$ satisfies

$$
J \subseteq J_{01}+J_{12}+\cdots+J_{n-1, n} .
$$

Therefore, $J^{n+1} \subseteq \sum_{0<i, j<n} J_{i j}^{2}$, and in fact we could have used the $n$th log infinitesimal neighborhood of the diagonal in place of $Z_{n}$. The same holds true for the global construction above. Now it is easy to see that the global construction reduces to the local construction of the last section in this case. From this we immediately see the equality of the five ideals.

Now to establish an isomorphism between $\prod_{j} \tilde{J}_{0 j}$ and $\Omega_{X / S}^{(n)}$, a similar proof to the proof of 1.5 shows that there are unique maps $\phi_{i}: \Omega_{X / S}^{1} \rightarrow \tilde{J}_{0 i}$ such that $\phi_{i}(d y)=$ $\pi_{i}^{*} y-\pi_{0}^{*} y$ for $y \in \mathscr{O}_{X}$, and $\phi_{i}(d \log m)=\alpha\left(\pi_{i}^{*} m-\pi_{0}^{*} m\right)-1$ for $m \in \mathscr{M}_{X}^{\mathrm{gp}}$. (Here, since $X \rightarrow \tilde{\Delta}_{X / S}^{n}$ is exact and $\pi_{i}^{*} m-\pi_{0}^{*} m$ pulls back to 0 in $\mathscr{M}_{X}^{\mathrm{gp}}$, we must have $\left.\pi_{i}^{*} m-\pi_{0}^{*} m \in \mathscr{M}_{\tilde{\Delta}_{X / S}^{n}}.\right)$ Therefore, there is a unique map $\Phi_{n}: \Omega_{X / S}^{(n)} \rightarrow \prod_{j=1}^{n} \tilde{J}_{0 j}$ such that

$$
\Phi_{n}\left(\omega_{1} \tilde{\wedge} \cdots \tilde{\wedge} \omega_{n}\right)=\phi_{1}\left(\omega_{1}\right) \cdots \phi_{n}\left(\omega_{n}\right)
$$

for $\omega_{1}, \ldots, \omega_{n} \in \Omega_{X / S}^{1}$. (The map exists locally by the previous section, and the uniqueness allows us to glue the local maps.) By the previous section, $\Phi_{n}$ is locally an isomorphism, so $\Phi_{n}$ gives a global isomorphism $\Omega_{X / S}^{(n)} \stackrel{\sim}{\rightarrow} \prod_{j=1}^{n} \tilde{J}_{0 j}$.

1.3. The Divided Power Envelope. In this section, let $D(n)$ denote the log PD envelope of the diagonal in $\Delta_{X / S}^{n}$. As before we get closed immersions $m_{i j}: D(n-$ $1) \rightarrow D(n)$. Let $\bar{J}_{i j} \subseteq \mathscr{O}_{D(n)}$ be the PD ideal corresponding to $m_{i j}, \Delta_{X / S}^{[n]}$ be the closed subscheme of $D(n)$ defined by $\sum_{i, j} \bar{J}_{i j}^{[2]}$, and $\tilde{J}_{i j}$ be the ideal of $\mathscr{O}_{\Delta_{X / S}^{[n]}}$ corresponding to $\bar{J}_{i j}$.

\section{Theorem 1.10.}

$$
\bigcap_{j=1}^{n} \tilde{J}_{0 j}=\prod_{j=1}^{n} \tilde{J}_{0 j}=\bigcap_{j=1}^{n} \tilde{J}_{j-1, j}=\prod_{j=1}^{n} \tilde{J}_{j-1, j}=\bigcap_{0 \leq i, j \leq n} \tilde{J}_{i j},
$$

and this ideal is canonically isomorphic to $\Omega_{X / S}^{n}$. 
Proof. First, observe that by the universal property of the PD envelope, $D(n) \simeq$ $D(1) \times_{X} \cdots \times_{X} D(1)$, the product of $n$ factors of $D(1)$, each considered as a scheme over $X$ via the projection $\pi_{0}$. Therefore, $\Delta_{X / S}^{[1]} \times_{X} \cdots \times_{X} \Delta_{X / S}^{[1]}$ is isomorphic to the closed subscheme of $D(n)$ corresponding to $\sum_{j} \bar{J}_{0 j}^{[2]}$. Also, it is easy to see that $\Delta_{X / S}^{[1]} \simeq \Delta_{X / S}^{(1)}$.

Therefore, to see that the previous map $\Psi_{n}$ induces a map $\mathscr{O}_{\Delta_{X / S}^{[n]}} \rightarrow \Omega_{X / S}^{n}$, it suffices to check that for $1 \leq i, j \leq n, \Psi_{n}\left[x \cdot\left(d^{i, j} y\right)^{[2]}\right]=\Psi_{n}\left[x \cdot\left(\delta^{i, j} p\right)^{[2]}\right]=0$ for $x \in \mathscr{O}_{D(n)}, y \in \mathscr{O}_{X}, p \in \mathscr{M}_{X}^{\mathrm{gp}}$. However, since $d^{i, j} y=d^{0, j} y-d^{0, i} y$, we have

$$
\begin{aligned}
\left(d^{i, j} y\right)^{[2]} & =\left(d^{0, j} y\right)^{[2]}+\left(d^{0, i} y\right)^{[2]}-\left(d^{0, i} y\right)\left(d^{0, j} y\right) \\
& \equiv-\left(d^{0, i} y\right)\left(d^{0, j} y\right) \quad\left(\bmod \bar{J}_{0 i}^{[2]}+\bar{J}_{0 j}^{[2]}\right) .
\end{aligned}
$$

Therefore,

$$
\begin{aligned}
\Psi_{n}\left(x \cdot\left(d^{i, j} y\right)^{[2]}\right) & =-\Psi_{n}\left(x\left(d^{0, i} y\right)\left(d^{0, j} y\right)\right) \\
& =(-1)^{i+j} d y \wedge d y \wedge \Psi_{n-2}\left(\mu_{0 i} \mu_{0 j} x\right)=0 .
\end{aligned}
$$

Similarly,

Therefore,

$$
\left(\delta^{i, j} p\right)^{[2]} \equiv-\left(\delta^{0, i} p\right)\left(\delta^{0, j} p\right) \quad\left(\bmod \bar{J}_{0 i}^{[2]}+\bar{J}_{0 j}^{[2]}\right)
$$

$$
\begin{aligned}
\Psi_{n}\left(x \cdot\left(\delta^{i, j} p\right)^{[2]}\right) & =-\Psi_{n}\left(x\left(\delta^{0, i} p\right)\left(\delta^{0, j} p\right)\right) \\
& =(-1)^{i+j} d \log p \wedge d \log p \wedge \Psi_{n-2}\left(\mu_{0 i} \mu_{0 j} x\right)=0 .
\end{aligned}
$$

Similarly, we already know $\Phi_{n}: \Omega_{X / S}^{(n)} \rightarrow \prod_{j=1}^{n} \tilde{J}_{0 j}$ is antisymmetric. Therefore, to check $\Phi_{n}$ induces a map $\Omega_{X / S}^{n} \rightarrow \prod_{j=1}^{n} \tilde{J}_{0 j}$, it suffices to check that it annihilates $d y \tilde{\wedge} d y \tilde{\wedge} \omega$ and $d \log p \tilde{\wedge} d \log p \tilde{\wedge} \omega$. However, from the above, we see that in fact

$$
\left(d^{0,1} y\right)\left(d^{0,2} y\right) \equiv-\left(d^{1,2} y\right)^{[2]} \quad\left(\bmod \bar{J}_{01}^{[2]}+\bar{J}_{02}^{[2]}\right),
$$

so $\phi_{1}(d y) \phi_{2}(d y) \in \bar{J}_{01}^{[2]}+\bar{J}_{02}^{[2]}+\bar{J}_{12}^{[2]}$, and this is zero in $\mathscr{O}_{\Delta_{X / S}^{[n]}}$. The proof that $\phi_{1}(d \log p) \phi_{2}(d \log p)=0$ in $\mathscr{O}_{\Delta_{X / S}^{[n]}}$ is similar.

Now since $\Psi_{n}$ and $\Phi_{n}$ were induced from inverse maps, they are inverse isomorphisms, and the equality of the ideals follows as before.

\section{The de Rham Complex}

We now describe the de Rham complex $\Omega_{X / S}$ in terms of our characterization of $\Omega_{X / S}^{n}$. We begin with the wedge product: thus, let $m, n>0$. Then we have a map

$$
\begin{aligned}
\Delta_{X / S}^{m+n} & \rightarrow \Delta_{X / S}^{m} \times_{X} \Delta_{X / S}^{n}, \\
\left(x_{0}, \ldots, x_{m}, \ldots, x_{m+n}\right) & \mapsto\left(\left(x_{0}, \ldots, x_{m}\right),\left(x_{m}, \ldots, x_{m+n}\right)\right) .
\end{aligned}
$$

Here we consider $\Delta_{X / S}^{m}$ as a scheme over $X$ via the last projection $\pi_{m}$, and $\Delta_{X / S}^{n}$ as a scheme over $X$ via the first projection $\pi_{0}$. This induces a map $D(m+n) \rightarrow$ $D(m) \times_{X} D(n)$, which in turn induces a map

$$
s_{m n}: \Delta_{X / S}^{[m+n]} \rightarrow \Delta_{X / S}^{[m]} \times_{X} \Delta_{X / S}^{[n]} .
$$


Locally, this map is also induced by the "smashing" map

$$
\begin{gathered}
B_{m}^{\prime} \otimes_{B} B_{n}^{\prime} \rightarrow B_{m+n}^{\prime}, \\
{\left[\left(y_{0} \otimes \cdots \otimes y_{m}\right) \otimes e^{\left(p_{0}, \ldots, p_{m}\right)}\right] \otimes\left[\left(y_{0}^{\prime} \otimes \cdots \otimes y_{n}^{\prime}\right) \otimes e^{\left(p_{0}^{\prime}, \ldots, p_{n}^{\prime}\right)}\right] \mapsto} \\
\left(y_{0} \otimes \cdots \otimes y_{m} y_{0}^{\prime} \otimes \cdots \otimes y_{n}^{\prime}\right) \otimes e^{\left(p_{0}, \ldots, p_{m}+p_{0}^{\prime}, \ldots, p_{n}^{\prime}\right)} .
\end{gathered}
$$

Remark 2.1. Although we also have a map $D(m) \times_{X} D(n) \rightarrow D(m+n)$, we do not get an induced map $\Delta_{X / S}^{[m]} \times_{X} \Delta_{X / S}^{[n]} \rightarrow \Delta_{X / S}^{[m+n]}$ in general. For example, the pullback of $\bar{J}_{0, m+n}^{[2]}$ does not correspond to anything from $\sum_{0 \leq i, j \leq m} \bar{J}_{i j}^{[2]}$ or $\sum_{0 \leq i, j \leq n} \bar{J}_{i j}^{[2]}$.

Proposition 2.2. We have a commutative diagram

$$
\begin{array}{ccc}
\bigcap_{i=1}^{m} \tilde{J}_{i-1, i} \otimes_{\mathscr{O}_{X}} \bigcap_{j=1}^{n} \tilde{J}_{j-1, j} & \stackrel{s_{m n}^{*}}{\longrightarrow} \bigcap_{j=1}^{m+n} \tilde{J}_{j-1, j} \\
\Psi_{m} \otimes \Psi_{n} \downarrow \simeq & & \Psi_{m+n} \downarrow \simeq \\
\Omega_{X / S}^{m} \otimes_{\mathscr{O}_{X}} \Omega_{X / S}^{n} & \stackrel{\wedge}{\longrightarrow} \Omega_{X / S}^{m+n} .
\end{array}
$$

Proof. Our first task is to verify that $s_{m n}^{*}$ actually induces a map as in the top row. To see this, note that

$$
s_{m n} \circ m_{j-1, j}= \begin{cases}\left(m_{j-1, j}, \mathrm{id}\right) \circ s_{m-1, n}, & j \leq m ; \\ \left(\mathrm{id}, m_{j-m-1, j-m}\right) \circ s_{m, n-1}, & j>m .\end{cases}
$$

Therefore, converting to dual statements in terms of $s_{m n}^{*}$ and $m_{j-1, j}^{*}$, we see that the image of $s_{m n}^{*}$ is annihilated by each $m_{j-1, j}^{*}$ and is thus in each kernel $\tilde{J}_{j-1, j}$.

Now to check the commutativity, we first reverse the vertical arrows and replace them by $\Phi_{m} \otimes \Phi_{n}$ and $\Phi_{m+n}$, respectively. Now, from the fact that

$$
\Phi_{n}\left(\omega_{1} \wedge \cdots \wedge \omega_{n}\right)=\psi_{1}\left(\omega_{1}\right) \cdots \psi_{n}\left(\omega_{n}\right),
$$

where $\psi_{j}(d y)=d^{j-1, j} y$ and $\psi_{j}(d \log p)=\delta^{j-1, j} p$, the commutativity is clear.

We now turn to the differential map in the de Rham complex; thus, fix $n \geq 0$. Then for $0 \leq j \leq n+1$, we have maps

$$
\Delta_{X / S}^{n+1} \rightarrow \Delta_{X / S}^{n},\left(x_{0}, \ldots, x_{j}, \ldots, x_{n+1}\right) \mapsto\left(x_{0}, \ldots, \hat{x}_{j}, \ldots, x_{n+1}\right) .
$$

These induce maps $D(n+1) \rightarrow D(n)$, which in turn induce maps

$$
d_{j}: \Delta_{X / S}^{[n+1]} \rightarrow \Delta_{X / S}^{[n]}
$$

Locally, these maps are also induced by the insertion maps

$$
\begin{aligned}
B_{n}^{\prime} & \rightarrow B_{n+1}^{\prime}, \\
\left(y_{0} \otimes \cdots \otimes y_{n}\right) \otimes e^{\left(p_{0}, \ldots, p_{n}\right)} & \mapsto\left(y_{0} \otimes \cdots \otimes 1 \otimes \cdots \otimes y_{n}\right) \otimes e^{\left(p_{0}, \ldots, 0, \ldots, p_{n}\right)},
\end{aligned}
$$

with insertion in the $j$ th position. 
Proposition 2.3. We have a commutative diagram

$$
\begin{array}{ccc}
\bigcap_{j=1}^{n} \tilde{J}_{j-1, j} & \stackrel{d_{0}^{*}-d_{1}^{*}+\cdots+(-1)^{n+1} d_{n+1}^{*}}{\longrightarrow} & \bigcap_{j=1}^{n+1} \tilde{J}_{j-1, j} \\
\Psi_{n}\lfloor\simeq & \Psi_{n+1} \mid \simeq \\
\Omega_{X / S}^{n} & \stackrel{d}{\longrightarrow} & \Omega_{X / S}^{n+1} .
\end{array}
$$

Proof. Let $e_{n}:=d_{0}^{*}-d_{1}^{*}+\cdots+(-1)^{n+1} d_{n+1}^{*}: \mathscr{O}_{\Delta_{X / S}^{[n]}} \rightarrow \mathscr{O}_{\Delta_{X / S}^{[n+1]}}$. Again, we first need to check that $e_{n}$ induces a map as in the top row. To see this, note that

$$
d_{j} \circ m_{i-1, i}= \begin{cases}m_{i-2, i-1} \circ d_{j}, & j<i-1 ; \\ \mathrm{id}, & j=i-1 \text { or } i \\ m_{i-1, i} \circ d_{j-1}, & j>i .\end{cases}
$$

From this, it is easy to check that the image of $e_{n}$ is annihilated by each $m_{i-1, i}^{*}$.

Now it follows formally from the appropriate identities that $e_{n+1} \circ e_{n}=0$, corresponding to the requirement that $d \circ d=0$. Furthermore,

$$
e_{m+n} \circ s_{m n}^{*}=s_{m+1, n}^{*} \circ\left(e_{m} \otimes \mathrm{id}\right)+(-1)^{m} s_{m, n+1}^{*} \circ\left(\mathrm{id} \otimes e_{n}\right),
$$

which corresponds to the requirement that $d(\omega \wedge \tau)=d \omega \wedge \tau+(-1)^{m} \omega \wedge d \tau$. It is easy to see that $e_{0}$ agrees with $d: \mathscr{O}_{X} \rightarrow \Omega_{X / S}^{1}$. Therefore, all that is left is to verify that $e_{1}(d \log p)=0$ for $p \in \mathscr{M}_{X}^{\mathrm{gp}}$. We calculate locally, where $d \log p=1 \otimes\left(e^{(-p, p)}-1\right) \in B_{1}^{\prime}$ for $p \in P^{\mathrm{gp}}$. Thus,

$$
e_{1}(d \log p)=1 \otimes e^{(0,-p, p)}-1 \otimes e^{(-p, 0, p)}+1 \otimes e^{(-p, p, 0)}-1 \otimes 1 .
$$

Now by the definition of $\Psi_{2}$, the last three terms are annihilated, and the first gets mapped to $-d \log p \wedge d \log p=0$.

Remark 2.4. In the case of trivial log structure, we have an easier proof: we see that $\Psi_{n+1}$ annihilates the image of $d_{j}^{*}$ for $j>0$, while locally,

$$
\Psi_{n+1}\left[d_{0}^{*}\left(y_{0} \otimes \cdots \otimes y_{n}\right)\right]=d y_{0} \wedge \cdots \wedge d y_{n}=d\left[\Psi_{n}\left(y_{0} \otimes \cdots \otimes y_{n}\right)\right] .
$$

However, to extend this proof to the case of $\log$ schemes, we must verify that

$$
\begin{aligned}
& d\left[y_{0} \alpha\left(p_{0}+\cdots+p_{n}\right)\left(d y_{1}+y_{1} d \log p_{1}\right) \wedge \cdots \wedge\left(d y_{n}+y_{n} d \log p_{n}\right)=\right. \\
& \alpha\left(p_{0}+\cdots+p_{n}\right)\left(d y_{0}+y_{0} d \log p_{0}\right) \wedge \cdots \wedge\left(d y_{n}+y_{n} d \log p_{n}\right) .
\end{aligned}
$$

While this can be done, we prefer to give the more conceptual proof above.

Remark 2.5. By taking the corresponding maps on the antisymmetric powers $\Omega_{X / S}^{(\cdot)}$ of $\Omega_{X / S}^{1}$, we can define a natural complex. However, from the above calculations, we see that we get $d(d \log m)=d \log m \tilde{\wedge} d \log m$, instead of 0 . This illustrates why in defining the logarithmic de Rham complex such that $d(d \log m)=0$, we need the full alternating product instead of just the antisymmetric product. (This requirement appears in the need to check that $d^{2} \alpha(m)=d(\alpha(m) d \log m)=0$.) 


\section{Coefficients}

In this section, we will assume that $X$ is $\log$ smooth over $S$, and $(E, \nabla)$ is an $\mathscr{O}_{X}$-module with quasi-nilpotent integrable connection. Then this corresponds to a crystal $E$ of $\mathscr{O}_{X / S}$-modules on the log crystalline site $(X / S)_{\text {cris }}$. Recall that an object of $(X / S)_{\text {cris }}$ is a tuple $(U, T, i, \delta)$ where $U$ is an open subscheme of $X, i: U \rightarrow T$ is an exact $\log$ closed immersion, and $\delta$ is a PD structure on the ideal of $i$. (For convenience of notation, we often use $T$ to represent this object.) Then a morphism $g: T_{1} \rightarrow T_{2}$ in this site is a morphism respecting the closed immersions and the PD structures, and a covering $\left(U_{\lambda}, T_{\lambda}, i_{\lambda}, \delta_{\lambda}\right)_{\lambda \in \Lambda}$ of $T$ is a family such that $\left(T_{\lambda}\right)$ is a Zariski open covering of $T$. Giving a sheaf $E$ on this site is then equivalent to giving a sheaf $E_{T}$ on $T$ for each object $T$ of $(X / S)_{\text {cris }}$, along with transition maps $\theta_{g}: g^{-1} E_{T_{2}} \rightarrow E_{T_{1}}$ for each morphism $g: T_{1} \rightarrow T_{2}$ in the site, satisfying the compatibility relation

$$
\theta_{h g}=\theta_{g} \circ g^{-1} \theta_{h}
$$

for the composition of $g: T_{1} \rightarrow T_{2}, h: T_{2} \rightarrow T_{3}$. We define $\mathscr{O}_{X / S}$ to be the sheaf with $\left(\mathscr{O}_{X / S}\right)_{T}:=\mathscr{O}_{T}$, and a sheaf $E$ of $\mathscr{O}_{X / S}$-modules is a crystal if for each morphism $g: T_{1} \rightarrow T_{2}$ in $(X / S)_{\text {cris }}$, the induced transition map $\theta_{g}: g^{*} E_{T_{2}} \rightarrow E_{T_{1}}$ is an isomorphism. For more details, see [2].

We note that by construction, each $\Delta_{X / S}^{[n]}$ is an object of $(X / S)_{\text {cris }}$, and each $d_{j}: \Delta_{X / S}^{[n+1]} \rightarrow \Delta_{X / S}^{[n]}$ is a morphism in this site. We thus get transition maps

$$
\theta_{d_{j}}: d_{j}^{*} E_{\Delta_{X / S}^{[n]}} \stackrel{\sim}{\rightarrow} E_{\Delta_{X / S}^{[n+1]}}
$$

Here we will consider $E_{\Delta_{X / S}^{[n]}}$ as being identified with $E \otimes_{\mathscr{O}_{X}} \mathscr{O}_{\Delta_{X / S}^{[n]}}$ via $\theta_{\pi_{0}}: \pi_{0}^{*} E_{X} \stackrel{\sim}{\rightarrow}$ $E_{\Delta_{X / S}^{[n]}}$.

Also, since the map

$$
\text { inc } \circ \Phi_{n}: \Omega_{X / S}^{n} \stackrel{\sim}{\rightarrow} \bigcap_{j=1}^{n} \tilde{J}_{j-1, j} \hookrightarrow \mathscr{O}_{\Delta_{X / S}^{[n]}}
$$

is a split injection (with splitting $\Psi_{n}$ ), so is the map id $\otimes\left(\right.$ inc $\left.\circ \Phi_{n}\right): E \otimes_{\mathscr{O}_{X}} \Omega_{X / S}^{n} \rightarrow$ $E \otimes_{\mathscr{O}_{X}} \mathscr{O}_{\Delta_{X / S}^{[n]}}$. Furthermore, we see that the image is equal to the intersection of the kernels of the transition maps $\theta_{m_{j-1, j}}: E_{\Delta_{X / S}^{[n]}} \rightarrow E_{\Delta_{X / S}^{[n+1]}}$. We will treat id $\otimes \Phi_{n}$ as identifying $E \otimes_{\mathscr{O}_{X}} \Omega_{X / S}^{n}$ with this submodule of $E \otimes_{\mathscr{O}_{X}} \mathscr{O}_{\Delta_{X / S}^{[n]}}$.

We now give a characterization of the de Rham complex with coefficients in $E$, in terms of the transition maps $\theta_{d_{j}}$ and the above identifications. 
Proposition 3.1. We have a commutative diagram

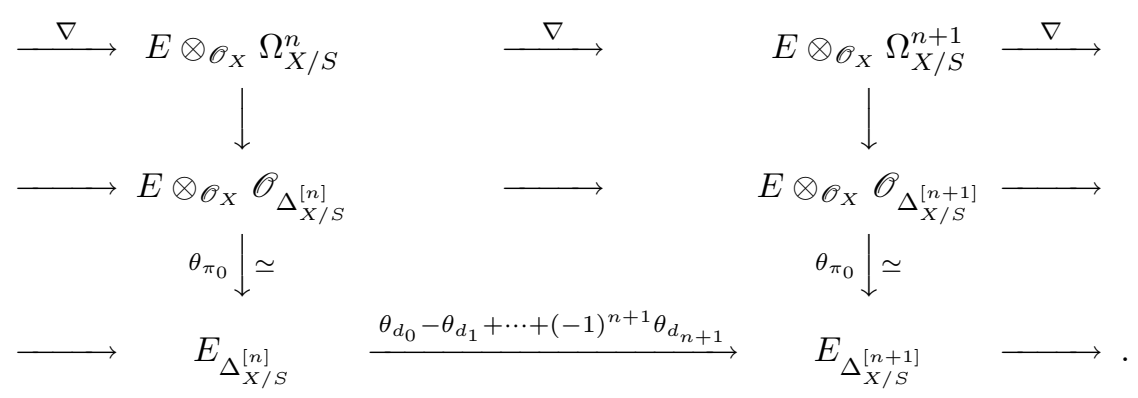

Proof. Let $e \in E, \omega \in \Omega_{X / S}^{n} \subseteq \mathscr{O}_{\Delta_{X / S}^{[n]}}$. If $j>0$, then since the composition of $d_{j}: \Delta_{X / S}^{[n+1]} \rightarrow \Delta_{X / S}^{[n]}$ and $\pi_{0}: \Delta_{X / S}^{[n]} \rightarrow X$ is equal to $\pi_{0}: \Delta_{X / S}^{[n+1]} \rightarrow X$, we see that $\theta_{d_{j}}(e \otimes \omega)=e \otimes d_{j}^{*} \omega$. Therefore, all we need to do to finish the proof is to show that $\theta_{d_{0}}(e \otimes \omega)=e \otimes d_{0}^{*} \omega+\theta_{s_{1 n}}(\nabla e \otimes \omega)$. Then since $\pi_{0} \circ s_{1 n}=\pi_{0}, \theta_{s_{1 n}}(\nabla e \otimes \omega)=\nabla e \wedge \omega$, and it immediately follows that for $\omega \in \Omega_{X / S}^{n}$,

$$
\left(\theta_{d_{0}}-\theta_{d_{1}}+\cdots \pm \theta_{d_{n+1}}\right)(e \otimes \omega)=\nabla e \wedge \omega+e \otimes d \omega=\nabla(e \otimes \omega) .
$$

However, by definition,

$$
\nabla=\theta_{\pi_{1}}-\theta_{\pi_{0}}: E \rightarrow E \otimes_{\mathscr{O}_{X}} \Omega_{X / S}^{1} \hookrightarrow E \otimes_{\mathscr{O}_{X}} \mathscr{O}_{\Delta_{X / S}^{[1]}} .
$$

Hence by the linearity of $\theta_{\pi_{1}}, \theta_{\pi_{1}}(e \otimes \omega)=e \otimes \omega+(\nabla e) \omega$ for $e \in E, \omega \in \mathscr{O}_{\Delta_{X / S}^{[1]}}$. Now considering the map $\left(\pi_{0}, \pi_{1}\right): \Delta_{X / S}^{[n]} \rightarrow \Delta_{X / S}^{[1]}$, we must have $\theta_{\pi_{1}}=\theta_{\left(\pi_{0}, \pi_{1}\right)} \circ$ $\left(\pi_{0}, \pi_{1}\right)^{*} \theta_{\pi_{1}}$, so

$$
\theta_{\pi_{1}}(e \otimes \omega)=e \otimes \omega+\left[\theta_{\left(\pi_{0}, \pi_{1}\right)}(\nabla e)\right] \omega
$$

for $e \in E, \omega \in \mathscr{O}_{\Delta_{X / S}^{[n]}}$. But since $\pi_{0} \circ d_{0}=\pi_{1}$, we now get

$$
\begin{aligned}
\theta_{d_{0}}(e \otimes \omega) & =\theta_{\pi_{1}}\left(e \otimes d_{0}^{*} \omega\right)=e \otimes d_{0}^{*} \omega+\left[\theta_{\left(\pi_{0}, \pi_{1}\right)}(\nabla e)\right] d_{0}^{*} \omega \\
& =e \otimes d_{0}^{*} \omega+\theta_{s_{1 n}}(\nabla e \otimes \omega) .
\end{aligned}
$$

Remark 3.2. It is easy to see, independently of the above calculation, that $\sum_{j}(-1)^{j} \theta_{d_{j}}$ induces maps $E \otimes_{\mathscr{O}_{X}} \Omega_{X / S}^{n} \rightarrow E \otimes_{\mathscr{O}_{X}} \Omega_{X / S}^{n+1}$ forming the differential maps in a complex. We thus have an alternate proof that the standard formula $\nabla(e \otimes \omega)=\nabla e \wedge \omega+e \otimes d \omega$ gives a well-defined complex, and in fact we see in this way that this is a natural generalization of the usual de Rham complex $\left(\Omega_{X / S}, d\right)$.

\section{Acknowledgements}

The author wishes to thank Lawrence Breen for inviting him to l'Université Paris 13 to give a talk on preliminary work on this subject, for useful discussions during that visit, and for providing comments on a draft version of this article.

The author has been supported by the research network Arithmetic Algebraic Geometry of the European Community (Contract MRTN-CT-2003-504917). 


\section{References}

[1] L. Breen and W. Messing, Combinatorial differential forms, Advances in Mathematics 164 (2001), no. 2, 203-282.

[2] K. Kato, Logarithmic Structures of Fontaine-Illusie, in J.-I. Igusa, editor, Algebraic Analysis, Geometry, and Number Theory, 191-224, The Johns Hopkins University Press (1988).

[3] A. Kock, Synthetic Differential Geometry, Vol. 51 of London Mathematical Society Lecture Note Series, Cambridge University Press (1981).

Scalable Network Technologies, 6100 Center Drive, Suite 1250, Los Angeles, CA 90045

E-mail address: schepler@math.berkeley.edu 